\title{
Advances in Diagnosis and Treatment of Cytomegalovirus Gastrointestinal Infection in Immunocompetent Adults
}

\author{
Guo Lin Dai, Chu Tian Wu, Shao Hui Tang* \\ The First Affiliated Hospital, Jinan University, Guangzhou, China \\ Email address: \\ tangshaohui206@163.com (Shao Hui Tang) \\ ${ }^{*}$ Corresponding author
}

To cite this article:

Guo Lin Dai, Chu Tian Wu, Shao Hui Tang. Advances in Diagnosis and Treatment of Cytomegalovirus Gastrointestinal Infection in Immunocompetent Adults. International Journal of Gastroenterology. Vol. 5, No. 1, 2021, pp. 18-25. doi: 10.11648/j.ijg.20210501.13

Received: January 4, 2021; Accepted: January 15, 2021; Published: January 22, 2021

\begin{abstract}
Cytomegalovirus (CMV), a kind of human herpes virus, is highly prevalent globally. It was believed that CMV posed a considerable risk only in newborns and immunocompromised individuals in the past, but there are increasing reports that CMV caused adverse outcomes in immunocompetent adults. The gastrointestinal canal (especially colon) is one of the usual sites of cytomegalovirus infection. Moreover, severe CMV gastrointestinal disease can lead to considerable mortality. Male, age $\geq 55$ years, immunomodulatory comorbidities (such as kidney failure, pregnancy, diabetes mellitus, or non-hematological malignancies without treatment) and the need for surgical intervention are factors that are not favorable to survival. When patients have unfavorable risk factors for survival or serious complications, antiviral treatment should be administered. Targeted antiviral therapy with intravenous ganciclovir or valganciclovir was suitable for severe CMV disease in immunocompetent adults before the advent of new and safer anti-herpesvirus drugs. Although there are many simple ways to detect the presence of $\mathrm{CMV}$, most of them cannot distinguish between latent infection and active disease, which makes clinical decision-making difficult. CMV gastrointestinal disease has no specific clinical and endoscopic manifestations, and the diagnosis is to a large extent dependent on histopathology and tissue CMV polymerase chain reaction technology. Quantitative testing of tissue CMV DNA is a useful diagnostic tool, but further research is needed to explain the quantitative value, such as obtaining a viral load cutoff to decide whether to apply antiviral therapy.
\end{abstract}

Keywords: Cytomegalovirus, Immunocompetent Adults, Gastrointestinal Infection, Diagnosis, Treatment

\section{Introduction}

Cytomegalovirus (CMV), a kind of human herpes virus, is highly prevalent globally. It may establish long-term latent latency, and can be reactivated to the host's immunity after the initial infection, rather than being completely eliminated. The gastrointestinal canal (especially colon) is one of the usual sites of CMV infection. Host immunity is the main determinant of the clinical manifestations of CMV disease. In the past, it was believed that CMV posed a considerable risk only in newborns and immunocompromised individuals [Solid organ or hematopoietic stem cell transplant recipients, patients with human immunodeficiency virus (HIV) infection and recipients of immunosuppressive drugs, including steroids or chemotherapeutic drugs], and it is often self-limiting or has minor clinical outcomes in immunocompetent adults [1-2]. However, there are increasing reports that CMV infects the gastrointestinal tract and causes adverse outcomes in immunocompetent adults. We ought to pay attention to this situation. Until now, there have been few studies on CMV gastrointestinal infections in immunocompetent adults, especially about the diagnosis and treatment. Also, there is much uncertainty because there are no standard patient management methods. This article reviews recent studies on CMV gastrointestinal infections in immunocompetent adults, especially in terms of risk factors and diagnosis and treatment, to help clinicians make accurate early diagnosis and improve patient prognosis.

\section{Epidemiology}

To date, there has been no complete statistical research about the incidence of CMV gastrointestinal infection in immunocompetent adults. Previous studies have shown that 
the infection of CMV is widespread among child-bearing age women, and the seroprevalence is between $45 \%$ and $100 \%$. CMV seroprevalence is higher in Asia, Africa and South America, and lower in the USA and Western Europe. Besides, CMV seroprevalence shows large geographic variations in the USA, which is up to $30 \%$ between states, but this could be explained by differences in the sample population. The seroprevalence among non-whites is often $20 \%$ to $30 \%$ higher than that of whites globally. Moreover, women and persons of lower socioeconomic status also have a higher CMV seroprevalence. Although the seroprevalence is high in some populations, a significant proportion of child-bearing age women are seronegative for $\mathrm{CMV}$ and therefore might be infected by CMV primarily during pregnancy, causing congenital CMV infection in infants [3]. There has been no epidemiological study on immunocompetent adults in the Middle East and North Africa. Among transplant recipients, CMV infection is common (as much as $77 \%$ ), but the true incidence of clinically diagnosed CMV disease is unknown because most researches have relied only on seropositivity reports. Therefore, there is an urgent need for elaborate studies within the World Health Organization (WHO) framework to assess the weight of CMV infection in immunocompetent populations in North Africa and the Middle East, predict the morbidity and mortality, and offer standardize treatment [4].

\section{Risk Factors}

In a retrospective study, Le et al. [5] divided CMV colitis patients into two groups: immunocompetent and immunocompromised, and there was no significant difference in inpatient mortality between them $(26.2 \%$ vs $25.9 \%)$. Unique significant independent predictive factor of inpatient mortality was to diagnose early, and the time limit for diagnosis was 9 days after admission. This means that CMV colitis in patients with immune function is more prevalent and lethal than previously recognized. Being aware that this disease may present in different ways will help to diagnose early and reduce mortality significantly. Galiatsatos et al. [6] reported the risk factors associated with mortality in immunocompetent patients with CMV colitis. Male, age $\geq 55$ years, immunomodulatory comorbidities (such as kidney failure, pregnancy, diabetes mellitus, or non-hematological malignancies without treatment) and the need for surgical intervention are factors that are not conducive to survival, and the mortality of patients with these factors is higher. On the contrary, healthy young patients get a favorable prognosis without intervention. However, Thanaboon Chaemsupaphan et al. [7] indicated that the immunocompetent adults who had CMV gastrointestinal diseases were mainly elderly, and the 6-mo mortality of immunocompetent patients was significantly higher $(39.0 \%$ vs. $22.0 \%)$. The independent predictive factors of death are advanced age, hospitalization, and the intensive care unit (ICU) clinical environment, and antiviral therapy is the only independent protective factor. Most of the immunocompetent patients are elderly and have accompanying by severe comorbidities, which may explains this. Among CMV colitis patients admitted to the ICU, despite ganciclovir treatment, the in-hospital mortality was still as high as $71.4 \%$ [8]. Additionally, pan colitis was also identified as a negative predictor of in-hospital mortality and overall survival rate [9].

A study conducted by Jae-Hoon Ko et al. [10] Showed that on univariate analysis, within 1 mo before diagnosis of CMV colitis, certain conditions including hemodialysis, neurological disease, rheumatism, admission of ICU, and use of steroids, antibiotics, antacids, or erythrocyte transfusions were all related to the occurrence of CMV colitis. Among them, use of steroid and erythrocyte transfusion within 1 mo were recognized to be independent risk factors in CMV colitis developing in immunocompetent hosts on multivariate analysis.

\section{Clinical Manifestations}

CMV infection among immunocompetent adults is generally symptomless or only causes slight mononucleosis-like syndrome, manifested by fever, fatigue, discomfort, lymphadenopathy, rash and other non-specific manifestations. CMV gastrointestinal infections can affect the whole gastrointestinal canal, and most commonly affect the colon. The incidence of severe CMV disease is higher than previously recognized, and it partly might be related to immune dysfunction caused by some comorbidities (e.g. diabetes and kidney disease) $[2,11]$.

Seo [12] and Siciliano [8] both showed that among immunocompetent patients, CMV colitis often occurs in aged patients with other comorbidities (such as chronic kidney disease, diabetes or ischemic cardiomyopathy), even in ICU patients. The most common symptom of these patients is gastrointestinal bleeding, and other symptoms like diarrhea, abdominal pain, bloating, nausea and vomiting can also be seen. Thus, we should be alert to such patients with gastrointestinal bleeding symptoms, which is probably the clinical manifestation of CMV colitis.

Compared with colitis, it is rare for CMV infection to manifest as proctitis [2]. Lee et al. [13] performed a retrospective analysis of 11 immunocompetent patients with CMV proctitis. They also found that the majority of patients were elder (average age 72 years), and the morbidity of diabetes was high $(54.5 \%)$. These patients mainly manifested with non-specific diarrhea, bloody stools, and tenesmus, and eight cases $(72.7 \%)$ were secondary to a life-threatening illness, such as cardiogenic shock or sepsis. Though the returns of antiviral therapy are controversial, clinicians ought to be alert that patients hospitalized for severe illness may possibally occur CMV proctitis reactivated.

CMV may also infect the upper gastrointestinal canal, of which the gastric antrum area is the most frequently infected site, followed by the cardia, esophagus, and duodenum. Patients with upper gastrointestinal infection mainly present with upper abdominal pain and upper gastrointestinal tract bleeding, nausea, vomiting and fever, occasionally chest discomfort and diarrhea, and patients with esophageal 
involved may also present with swallowing pain [14-16].

In addition to the above clinical manifestations, CMV-induced pseudotumor is seen occasionally. Jacob et al. [17] reported a rare case with an ulcerative polypoid mass in the anorectal area. Pathological biopsy showed large intrabasal nucleus inclusions, and they were positive for CMV in immunohistochemical staining. Two weeks of antiviral therapy was effective for the patient, and the mass eventually subsided completely.

\section{Complications}

CMV gastrointestinal infection also brings a series of serious life-threatening complications, such as septicemia secondary to colitis, lower gastrointestinal bleeding, ischemic enteritis, toxic megacolon, and colon perforation [18-22]. Acute CMV infection can also be complicated by venous thrombosis, but the relation between arterial thrombosis and CMV infection is not yet clear [23]. Atzmony et al. [24] researched the morbidity of thrombosis in inpatients with acute CMV infection retrospectively, and the morbidity was approximately $6.4 \%$. But it is still unknow in patients with intestinal CMV infections. According to Justo et al. [23], visceral venous thrombosis (splenic vein) is the most common among immunocompetent patients with CMV infections, followed by deep vein thrombosis and pulmonary embolism, while it is exactly the opposite among immunocompromised patients. In addition, although isolated CMV gastrointestinal infections can often be relieved with fewer complications or even self-healing in young immunocompetent patients, some patients will still be diagnosed with inflammatory bowel disease (IBD) subsequently [25].

\section{Diagnoses}

\subsection{Endoscopy}

Endoscopic manifestations of CMV gastrointestinal infection are non-specific, and can be manifested as mucosal erythema, ulcers, and mucosal thickening [14]. Puo-hsien et al. [9] retrospectively analyzed non-IBD patients with CMV colitis, and ulcers (77\%) were the commonest endoscopic manifestation, following pebble-like appearance (19.7\%), colon inflammation whether with erosion or not $(9.8 \%)$, pseudomembranous inflammation $(9.8 \%)$ as well as tumor-like lesions $(8.2 \%)$. Ulcers wrapped in albuginea and typical deep ulcers are more representative in CMV colitis. Among patients undergoing colonoscopy, right-sided, left-sided and pan colitis accounted for $35.3 \%, 23.5 \%$ and $32.4 \%$ respectively, and pan colitis can be a negative factor for in-hospital mortality and survival rate. Therefore, colonoscopy can be used for accurate diagnosis and predict the prognosis of CMV colitis patients without IBD. Since the presentation of CMV colitis is non-specific, multiple random biopsies are supposed to be performed at the lesion in appropriate clinical circumstances, regardless of the performance of colonoscopy. Endoscopists should take biopsy samples from the inflammatory mucosa near or inside the ulcer and from adjoining normal mucosa when they see inflammatory mucosa. [26]. As for the most common site of CMV colitis, there is no definite conclusion.

In the upper gastrointestinal CMV disease, a retrospective study [14] pointed out that endoscopically examined mucosa showed fragile congestion or obvious erythema. Eleven cases (37\%) showed single ulcers, and ulcers of the gastric pylorus and cardia were larger than those of other parts, with a diameter $>2 \mathrm{~cm}$. Eight cases $(27 \%)$ showed multiple ulcers, and the number of ulcers was 2-6 with a diameter from 0.6 to $0.8 \mathrm{~cm}$. Thirteen cases (43\%) showed mucosal thickening (including localized thickening, polyps or fold hypertrophy), which could only be seen in the gastric area. Necrotic substances and granulation tissue with moderate to severe lymphoplasma cell infiltration and gastric foveola hyperplasia can be seen in ulcerative lesions under the light microscope, with mild to moderate chronic inflammatory infiltration and gastric foveola hyperplasia in mucosal thickening lesions.

That CMV gastrointestinal infection manifests as isolated tumor-like or polypoid-like lesions [14, 17, 27] should draw our attention. These reports expand the scope of endoscopic and histological representation of CMV gastrointestinal infection, and we should strengthen our ability to detect and classify all these CMV disease types that may not be fully acknowledged. It should be emphasized that in the absence of obvious gastrointestinal symptoms, routine colonoscopy is not mandatory for CMV disease diagnosis. In particular, when antiviral drugs are still the main treatment, endoscopic examination will not change the overall management much, but it will expose patients to unnecessary endoscopic risks [28].

\subsection{Antibodies}

In primary infections, IgM antibodies appear and increase early and could be tested within $1 \mathrm{wk}$ after infection with $100 \%$ sensitivity and 99\% specificity. Over the subsequent 3-6 mo, IgM becomes undetectable. The production of IgG antibodies occurs within a few weeks after IgM elevation, and elevated levels are maintained for a long time to provide lifetime protection. Patients are thought to be seropositive if CMV-specific IgG antibodies are detected. During the process of CMV reactivation, the IgG antibody titer doesn't change, and the reactivation can be distinguished only by testing $\operatorname{IgM}$ antibody titer increases, which doesn't occur always, and is usually delayed or even absent in reactivation as well as reinfection. Therefore, CMV antibodies are limited in the diagnosis of clinical CMV reactivation [29].

\subsection{Antigen}

Eguchi et al. [30] evaluated the accuracy of detecting CMV antigenemia (PP65 antigen) to diagnose CMV diseases confirmed by real-time quantitative PCR (qPCR). They found that the pooled estimates of sensitivity and specificity of PP65 antigen to diagnose CMV infection were 0.65 and 0.94 respectively. That is to say, pp65 has a good positive 
predictive value in the diagnosis of CMV infection. However, it also means that $35 \%$ of patients with CMV infection confirmed by real-time qPCR have negative antigenemia. Therefore, negative results for antigenemia testing cannot exclude CMV infection. A recent retrospective study ${ }^{31}$ found that the sensitivity and specificity of the peripheral blood pp65 antigen immunofluorescence assay were $50 \%$ and $100 \%$ respectively, which was similar to the results of Eguchi et al. This method is simple and fast, but it cannot be use to distinguish between potential infection and active disease.

\subsection{Histopathology}

Histopathological examination mainly includes CMV-specific immunohistochemical and hematoxyli-eosin (HE) staining. Immunohistochemical labeling of CMV antigen in infected gastrointestinal mucosa cells is thought as the gold standard for diagnosis of CMV gastrointestinal infections. The sensitivity and specificity of HE staining were $17 \%$ and $98 \%$ respectively, and immunohistochemistry was $67 \%$ and $98 \%$ [31]. In HE staining of tissue sections, it can be seen that the nuclei involved in CMV are significantly increased, up to 3-4 times the size of lymphocyte nuclei. The nuclei are basophilic, with uniformly dark-stained or cloud-like CMV inclusions. Sometimes the inclusions are small and there is a clear halo around them, which looks like an "eagle eye". Sometimes they are elongated in the smooth-muscle cell nuclei. Infected cells are most commonly found in endothelial cells in granulation tissue, and sometimes in smooth-muscle cells and epithelial cells. Immunohistochemistry shows that the nuclei involved in CMV infection are uniformly dark-stained tan nuclei, sometimes with diffuse nuclei and cytoplasmic staining [32]. Although histopathological examination has high specificity, it is invasive, has low sensitivity and thus somehow depends on the operator to avoid sampling errors [33]. Clinical suspicion for CMV disease has high returns for CMV detection, but upfront testing may be unnecessary for the economy and invasiveness of immunohistochemistry. Careful histopathological review by pathologists is of great importance to detect CMV efficiently and cost-effectively [34].

\subsection{PCR}

The status of histopathology is unquestionable, but the detection of CMV DNA in gastrointestinal biopsy specimen by real-time qPCR is a sensitive, objective, rapid, and highly standardized diagnostic method. If the histopathology is negative, CMV diseases ought to be diagnosed with real-time qPCR virus detection based on clinical background (such as symptoms, endoscopic findings, and no other infections) [35]. Kredel et al. [31] found that the sensitivity of tissue PCR was $100 \%$ and specificity was $94 \%$. Tsuchido et al. [36] calculated that the sensitivity of $\mathrm{qPCR}$ in the population of non-HIV immunocompromised patients was $89.3 \%$ and specificity was $85.6 \%$ by receiver operating characteristic curve (ROC) at a critical value of 180 copies $/ \mu \mathrm{g}$ DNA. This confirms the good diagnostic performance of qPCR based on endoscopic biopsy to detect CMV disease in non-HIV immunocompromised patients. Moreover, several studies [36-38] showed that real-time qPCR is both sensitive and specific for $\mathrm{CMV}$ detection in tissue of formalin fixed and paraffin embedded, and it can be complementary to conventional histopathology (especially immunohistochemistry), because $10 \%$ to $15 \%$ of the missed diagnoses by one method could be tested by the other method. Therefore, real-time qPCR should be used for cases with high clinical suspicion but cannot be diagnosed by immunohistochemistry. However, it should be noted that CMV may still be lurking in white blood cells after early infection. That is to say, because of the high sensitivity of real-time qPCR detection, a positive result doesn't mean to be an active infection.

Kredel et al. [31] pointed out that if we use clinical parameters like aleucemia, splenomegalia, and steroid-refractory disease to detect CMV colitis, negative result of blood-based CMV PCR can rule out CMV colitis, while a $75 \%$ chance of positive result to develop clinically related CMV colitis. This indicates a good specificity that blood-based CMV PCR combined with convenient clinical parameters are able to rule out clinically related CMV colitis. The results of plasma-based CMV PCR wrer compared by Lisboa et al. [39] with whole blood samples, and their ability to predict relapse was evaluated. They found that whole blood PCR was more sensitive because it could detect viruses inside and outside the cells at the same time, but it doesn't appear to provide significant clinical returns, nor dose it better predict CMV disease recurrence.

\subsection{Virus Culture}

Although virus culture has high specificity, it has been replaced clinically by histopathology and tissue PCR technology because of its low sensitivity and time-consuming performance [40].

\subsection{Feces}

Fecal CMV real-time PCR is a newer detection technology that can be applied to diagnose CMV gastrointestinal diseases as a non-invasive way. An earlier retrospective study [41] showed that negative results for stool CMV PCR cannot exclude CMV gastrointestinal diseases because of the low sensitivity of $67 \%$, and CMV diseases must be confirmed by gastrointestinal biopsy. However, positive results have specificity of up to $96 \%$, which plays a important role in diagnosis and helps to avoid invasive diagnostic procedures, such as endoscopy and histopathology. Recently, Prachasitthisak et al. [42] pointed out that fecal CMV real-time PCR has a sensitivity of $71 \%$, specificity of $85 \%$ and accuracy of $82 \%$, and there was a significant correlation between fecal and plasma CMV real-time PCR. This indicates a high diagnostic value, which is similar to the results of previous research. 


\section{Differential Diagnosis}

The clinical and endoscopic manifestations of CMV gastrointestinal diseases are not specific, which can lead to many differential diagnoses. For example, the differential diagnosis of gastrointestinal bleeding includes diverticulosis, ischemic colitis, postoperative bleeding, Dieulafoy's disease, IBD, colorectal polyps or tumor, and anorectal disease [43]. Ulcers caused by CMV gastrointestinal diseases can also be caused by other infectious diseases, such as enteritidis of Shigella, Salmonella, Clostridium difficile as well as staphylococcus, tuberculous, and even parasites. Others include gastrointestinal lupus vasculitis, actinomycosis, lymphoma and amoebiasis [44]. It is particularly vital that CMV colitis should not be misdiagnosed as new IBD among these differential diagnoses, because the main treatment for IBD is the application of immunosuppressive drugs, which is harmful to control CMV disease.

\section{Treatment and Prognosis}

First, we need to differentiate CMV infection from CMV disease. CMV infection means that the virus can be detected in the human body, while CMV disease is related to the symptoms caused by the virus. Although first-line drugs advised for severe CMV disease treatment in immunocompromised people are valganciclovir or ganciclovir currently [45], few researches assessed these drugs for CMV disease treatment in immunocompetent individuals. Some literature $[15,46]$ points out that asymptomatic and mild infections of immunocompetent individuals do not require antiviral treatment, and it is recommended to guide treatment by combining disease severity and viral load.

However, severe untreated CMV disease leads to high mortality. Despite little evidence, targeted antiviral use of intravenous valganciclovir or ganciclovir is suitable for immunocompetent adults with severe CMV disease before the advent of new and safer anti-herpesvirus drugs. Although the clinical symptoms are obvious, CMV colitis has a favorable prognosis if diagnosed and treated early. Foscarnet and cidofovir have worse adverse effects, and they are only used when there is resistance to ganciclovir [11-12, 47-48]. These antiviral drugs all have many adverse effects, and serious ones include bone marrow suppression, potential carcinogenicity, central nervous system disorders, liver toxicity and nephrotoxicity. The specific pharmacologic action and side effects of the drugs are shown in Table 1 [20, 33, 49, 50].

Table 1. Pharmacologic Action and Side Effects of Antiviral Drugs.

\begin{tabular}{lll}
\hline Drugs & Pharmacologic Action & Side Effects \\
\hline Ganciclovir & $\begin{array}{l}\text { Competitively inhibits dGTP to combine with DNA } \\
\text { polymerase, thereby inhibiting the synthesis of viral DNA. } \\
\text { Converted to ganciclovir, and has a better bioavailability } \\
\text { Valganciclovir }\end{array}$ & $\begin{array}{l}\text { Hepatotoxicity, nephrotoxicity, neurotoxicity, neutropenia, leukopenia, } \\
\text { anemia and thrombocytopenia, potential carcinogenicity }\end{array}$ \\
Cidofovir & $\begin{array}{l}\text { Suppresses replication of CMV through selectively inhibiting } \\
\text { viral DNA synthesis }\end{array}$ & $\begin{array}{l}\text { Ocular toxicity, nephrotoxicity, gastrointestinal toxicity, fever, hair loss, } \\
\text { rash, cough, dyspnea }\end{array}$ \\
Foscarnet & $\begin{array}{l}\text { Non-competitive inhibitor of various viral RNA and DNA } \\
\text { polymerases }\end{array}$ & $\begin{array}{l}\text { Electrolyte imbalance, diarrhea, vomiting, fever, anemia, neutropenia, } \\
\text { nephrotoxicity, neurotoxicity, cardiotoxicity, hepatotoxicity }\end{array}$ \\
\hline
\end{tabular}

In contrast, two randomized controlled trials [51, 52], indicated that ganciclovir or valganciclovir used for CMV disease treatment in critically ill patients can significantly inhibit CMV reactivation, but neither has been found to reduce mortality and the length of hospital stay. In another retrospective study [47], 36 patients received antiviral therapy. Two patients received antiviral therapy died, and all others who didn't receive the therapy recovered spontaneously, indicating that antiviral therapy ought not to be compulsive for some CMV colitis patients.

Therefore, at present, most researchers endorse that when patients have risk factors that are unfavorable for survival, including male, age $\geq 55$ years, immunomodulatory comorbidities (such as kidney failure, pregnancy, diabetes mellitus, or non-hematological malignancies without treatment) [6], or when serious complications like gastrointestinal bleeding, intestinal perforation, or toxic megacolon occur, antiviral therapy is recommended [19, 53, 54].

\section{Conclusion and Outlook}

To date, there has been no complete epidemiological study on the statistics of the incidence of CMV gastrointestinal infections in immunocompetent adults, especially in the Middle East and North Africa. Therefore, there is an urgent need for elaborate studies within the World Health Organization (WHO) framework to assess the weight of CMV infection in immunocompetent people, so we can predict their morbidity and mortality accurately, and carry out prevention and standardized treatment. Additionally, child-bearing age women might be infected by CMV primarily during pregnancy, which will result in congenital CMV infection in their infants. Therefore, it may be necessary to take actions to protect this population in the future, such as developing vaccines to prevent primary infections of pregnant women, researching and developing new drugs to cut off the mother-to-child transmission of CMV and carrying out health promotion activities to lower risks of congenital CMV infection.

CMV colitis in immunocompetent people is not common, but its mortality is high. It ought to be one of the differential diagnoses for colitis patients without immunodeficiency but with risk factors like chronic kidney disease, diabetes, and advanced age. There is a low incidence of severe CMV diseases, non-specific clinical and endoscopic manifestations and some of their misleading manifestations (such as polypoid masses), and unconventional histopathology and tissue CMV 
PCR technology that diagnosis depends on. This requires clinicians to be more vigilant and ask pathologists to carry out detailed histopathology and tissue PCR examinations to improve the sensitivity of testing. Therefore, for the purpose of reducing mortality and morbidity, clinicians can make early diagnosis and treatment.

Many simple methods can be used to test the existence of CMV currently, even to quantify viral lord, but the current blood viral load test is less specific in detecting CMV gastrointestinal diseases. Moreover, a "gold standard" that can easily distinguish between latent infection and active disease is lack, which makes clinical decision-making difficult. Future research may investigate the predictive value of positive viral load in patients with gastrointestinal symptoms. Quantitative testing of tissue CMV DNA is a useful diagnostic tool, but further research is needed to explain the quantitative value, such as obtaining a viral load cutoff to decide whether to apply antiviral therapy. In the future, histological viral load testing is expected to become the gold standard to diagnose CMV colitis.

Follow-up can find some CMV colitis patients develop IBD, mainly manifesting with abdominal pain and diarrhea. CMV infection may be considered as the cause of IBD. At present, the pathogenesis of intestinal diseases caused by CMV infection is still unclear. It is necessary to conduct further studies about the pathogenic role and mechanism of CMV in intestinal diseases and its possible relationship with IBD, to provide reliable evidence for optimizing clinical diagnosis and treatment.

Current drugs used to treat CMV disease have serious adverse effects, and CMV may develop resistance to these drugs. Fecal microbiota transplantation is a new treatment for some gastrointestinal diseases, and its safety and efficacy for CMV gastrointestinal diseases needs to be further studied.

\section{Author Contributions}

Guo-Lin Dai wrote the paper. Chu-Tian Wu participated in reviewing literature addressing CMV diagnosis and participated in drafting the manuscript. All authors read and approved the final manuscript.

\section{Conflict of Interest}

The authors declare that they have no conflict of interest.

\section{Acknowledgements}

We would like to thank all the authors who had participated in this review. This review received no specific grant from any funding agency in the public, commercial, or not-for-profit sectors.

\section{References}

[1] Griffiths P, Baraniak I, Reeves M. The pathogenesis of human cytomegalovirus. J Pathol. 2015; 235: 288-97.
[2] Rafailidis PI, Mourtzoukou EG, Varbobitis IC, et al. Severe cytomegalovirus infection in apparently immunocompetent patients: a systematic review. Virol J. 2008; 5: 47.

[3] Cannon MJ, Schmid DS, Hyde TB. Review of cytomegalovirus seroprevalence and demographic characteristics associated with infection. Reviews in Medical Virology. 2010; 20: 202-13.

[4] Al Mana H, Yassine HM, Younes NN, et al. The Current Status of Cytomegalovirus (CMV) Prevalence in the MENA Region: A Systematic Review. Pathogens. 2019; 8.

[5] Le PH, Lin WR, Kuo CJ, et al. Clinical characteristics of cytomegalovirus colitis: a 15-year experience from a tertiary reference center. Ther Clin Risk Manag. 2017; 13: 1585-93.

[6] Galiatsatos P, Shrier I, Lamoureux E, et al. Meta-analysis of outcome of cytomegalovirus colitis in immunocompetent hosts. Dig Dis Sci. 2005; 50: 609-16.

[7] Chaemsupaphan T, Limsrivilai J, Thongdee C, et al. Patient characteristics, clinical manifestations, prognosis, and factors associated with gastrointestinal cytomegalovirus infection in immunocompetent patients. BMC Gastroenterol. 2020; 20: 22.

[8] Siciliano RF, Castelli JB, Randi BA, et al. Cytomegalovirus colitis in immunocompetent critically ill patients. Int $\mathrm{J}$ Infect Dis. 2014; 20: 71-3.

[9] Le PH, Kuo CJ, Wu RC, et al. Pancolitis associated with higher mortality risk of cytomegalovirus colitis in patients without inflammatory bowel disease. Ther Clin Risk Manag. 2018; 14: 1445-51.

[10] Ko JH, Peck KR, Lee WJ, et al. Clinical presentation and risk factors for cytomegalovirus colitis in immunocompetent adult patients. Clin Infect Dis. 2015; 60: e20-6.

[11] Lancini D, Faddy HM, Flower R, et al. Cytomegalovirus disease in immunocompetent adults. Med J Aust. 2014; 201: 578-80.

[12] Seo TH, Kim JH, Ko SY, et al. Cytomegalovirus colitis in immunocompetent patients: a clinical and endoscopic study. Hepatogastroenterology. 2012; 59: 2137-41.

[13] Lee CY, Chen YH, Lu PL. Reactivated cytomegalovirus proctitis in an immunocompetent patient presenting as nosocomial diarrhea: a case report and literature review. BMC Infect Dis. 2017; 17: 113.

[14] Reggiani Bonetti L, Losi L, Di Gregorio C, et al. Cytomegalovirus infection of the upper gastrointestinal tract: a clinical and pathological study of 30 cases. Scandinavian Journal of Gastroenterology. 2011; 46: 1228-35.

[15] Gravito-Soares E, Gravito-Soares M, Camacho E, et al. Cytomegalovirus ulcerative oesophagitis in a young healthy immunocompetent patient. 2018; 2018: bcr-2017-223297.

[16] Costa D, Fernandes D, Furtado A, et al. Cytomegalovirus duodenitis in immunocompetent patients: what else should we look for? BMJ Case Rep. 2017; 2017.

[17] Jacob S, Zayyani N. Cytomegalovirus colitis masquerading as rectal malignancy in an immunocompetent patient. Indian Journal of Pathology and Microbiology. 2015; 58: 80-2.

[18] D'cruz RT, Lau CC-L, Thamboo TP. Severe ischemic cytomegalovirus proctocolitis with multiple perforation. Archives of Virology. 2018; 163: 1927-31. 
[19] Chan KS, Lee WY, Yu WL. Coexisting cytomegalovirus infection in immunocompetent patients with Clostridium difficile colitis. J Microbiol Immunol Infect. 2016; 49: 829-36.

[20] Hasegawa T, Aomatsu K, Nakamura M, et al. Cytomegalovirus colitis followed by ischemic colitis in a non-immunocompromised adult: a case report. World J Gastroenterol. 2015; 21:3750-4.

[21] Lin YH, Yeh CJ, Chen YJ, et al. Recurrent cytomegalovirus colitis with megacolon in an immunocompetent elderly man. Journal of medical virology. 2010; 82: 638-41.

[22] Cho JH, Choi JH. Cytomegalovirus ileo-pancolitis presenting as toxic megacolon in an immunocompetent patient: A case report. World journal of clinical cases. 2020; 8: 552-9.

[23] Justo D, Finn T, Atzmony L, et al. Thrombosis associated with acute cytomegalovirus infection: a meta-analysis. European journal of internal medicine. 2011; 22: 195-9.

[24] Atzmony L, Halutz O, Avidor B, et al. Incidence of cytomegalovirus-associated thrombosis and its risk factors: a case-control study. Thrombosis research. 2010; 126: e439-43.

[25] Khan TV, Toms C. Cytomegalovirus Colitis and Subsequent New Diagnosis of Inflammatory Bowel Disease in an Immunocompetent Host: A Case Study and Literature Review. Am J Case Rep. 2016; 17: 538-43.

[26] Garrido E, Carrera E, Manzano R, et al. Clinical significance of cytomegalovirus infection in patients with inflammatory bowel disease. World J Gastroenterol. 2013; 19: 17-25.

[27] Agaimy A, Mudter J, Märkl B, et al. Cytomegalovirus infection presenting as isolated inflammatory polyps of the gastrointestinal tract. Pathology. 2011; 43: 440-6.

[28] Wong YJ. Cytomegalovirus infection in an immunocompetent patient. Gastrointestinal Endoscopy. 2018; 88: 969-70.

[29] Baniak N, Kanthan R. Cytomegalovirus Colitis: An Uncommon Mimicker of Common Colitides. Arch Pathol Lab Med. 2016; 140: 854-8.

[30] Eguchi H, Horita N, Ushio R, et al. Diagnostic test accuracy of antigenaemia assay for PCR-proven cytomegalovirus infection-systematic review and meta-analysis. Clin Microbiol Infect. 2017; 23: 907-15.

[31] Kredel LI, Mundt P, van Riesen L, et al. Accuracy of diagnostic tests and a new algorithm for diagnosing cytomegalovirus colitis in inflammatory bowel diseases: a diagnostic study. International Journal of Colorectal Disease. 2019; 34: 229-37.

[32] Zhou WX, Yang H, Ling Q, et al. Histologic detection of cytomegalovirus and its application in gastrointestinal cytomegalovirus infection. Chin J Pathol. 2016; 0.

[33] Al-Omari A, Aljamaan F, Alhazzani W, et al. Cytomegalovirus infection in immunocompetent critically ill adults: literature review. Ann Intensive Care. 2016; 6: 110.

[34] Juric-Sekhar G, Upton MP, Swanson PE, et al. Cytomegalovirus (CMV) in gastrointestinal mucosal biopsies: should a pathologist perform CMV immunohistochemistry if the clinician requests it? Human Pathology. 2017; 60: 11-5.

[35] Bernard S, Germi R, Lupo J, et al. Symptomatic cytomegalovirus gastrointestinal infection with positive quantitative real-time PCR findings in apparently immunocompetent patients: a case series. Clin Microbiol Infect. 2015; 21: 1121 e1-7.

[36] Tsuchido $Y$, Nagao $M$, Matsuura $M$, et al. Real-time quantitative PCR analysis of endoscopic biopsies for diagnosing CMV gastrointestinal disease in non-HIV immunocompromised patients: a diagnostic accuracy study. European Journal of Clinical Microbiology \& Infectious Diseases. 2018; 37: 2389-96.

[37] Mills AM, Guo FP, Copland AP, et al. A Comparison of CMV Detection in Gastrointestinal Mucosal Biopsies Using Immunohistochemistry and PCR Performed on Formalin-fixed, Paraffin-embedded Tissue. 2013; 37: 995-1000.

[38] McCoy MH, Post K, Sen JD, et al. qPCR increases sensitivity to detect cytomegalovirus in formalin-fixed, paraffin-embedded tissue of gastrointestinal biopsies. Hum Pathol. 2014; 45: 48-53.

[39] Lisboa LF, Asberg A, Kumar D, et al, Preiksaitis JK, et al. The clinical utility of whole blood versus plasma cytomegalovirus viral load assays for monitoring therapeutic response. Transplantation. 2011; 91: 231-6.

[40] Osawa R, Singh N. Cytomegalovirus infection in critically ill patients: a systematic review. Crit Care. 2009; 13: R68.

[41] Ganzenmueller T, Kluba J, Becker JU, et al. Detection of cytomegalovirus (CMV) by real-time PCR in fecal samples for the non-invasive diagnosis of CMV intestinal disease. J Clin Virol. 2014; 61: 517-22.

[42] Prachasitthisak N, Tanpowpong P, Lertudomphonwanit C, et al Short article: Stool cytomegalovirus polymerase chain reaction for the diagnosis of cytomegalovirus-related gastrointestinal disease. Eur J Gastroenterol Hepatol. 2017; 29: 1059-63.

[43] Strate LL, Gralnek IM. ACG Clinical Guideline: Management of Patients with Acute Lower Gastrointestinal Bleeding. Am J Gastroenterol. 2016; 111: 459-74.

[44] Seminari E, Fronti E, Contardi G, et al. Colitis in an elderly immunocompetent patient. Journal of Clinical Virology. 2012; 55: $187-90$.

[45] Jones A, McCurdy JD, Loftus EV, et al. Effects of antiviral therapy for patients with inflammatory bowel disease and a positive intestinal biopsy for cytomegalovirus. Clinical gastroenterology and hepatology: the official clinical practice journal of the American Gastroenterological Association. 2015; 13: 949-55.

[46] Li FR, Gong YN, Li J. [Cytomegalovirus enteritis in an immunocompetent child]. Zhonghua Er Ke Za Zhi. 2018; 56: 784-5.

[47] Kim CH, Bahng S, Kang KJ, et al. Cytomegalovirus colitis in patients without inflammatory bowel disease: a single center study. Scand J Gastroenterol. 2010; 45: 1295-301.

[48] Forel JM, Martin-Loeches I, Luyt CE. Treating HSV and CMV reactivations in critically ill patients who are not immunocompromised: pro. Intensive Care Med. 2014; 40: 1945-9.

[49] Mareri A, Lasorella S, Iapadre G, et al. Anti-viral therapy for congenital cytomegalovirus infection: pharmacokinetics, efficacy and side effects. J Matern Fetal Neonatal Med. 2016; 29: 1657-64. 
[50] Ritchie BM, Barreto JN, Barreto EF, et al. Relationship of Ganciclovir Therapeutic Drug Monitoring with Clinical Efficacy and Patient Safety. Antimicrobial agents and chemotherapy. 2019; 63.

[51] Cowley NJ, Owen A, Shiels SC, et al. Safety and Efficacy of Antiviral Therapy for Prevention of Cytomegalovirus Reactivation in Immunocompetent Critically Ill Patients: A Randomized Clinical Trial. JAMA Intern Med. 2017; 177: 774-83.

[52] Limaye AP, Stapleton RD, Peng L, et al. Effect of Ganciclovir on IL-6 Levels Among Cytomegalovirus-Seropositive Adults With Critical Illness: A Randomized Clinical Trial. JAMA. 2017; 318: 731-40.

[53] Fyock C, Gaitanis M, Gao J, et al. Gastrointestinal CMV in an elderly, immunocompetent patient. Rhode Island medical journal (2013). 2014; 97: 53-6.

[54] Harano Y, Kotajima L, Arioka H. Case of cytomegalovirus colitis in an immunocompetent patient: a rare cause of abdominal pain and diarrhea in the elderly. Int J Gen Med. 2015; 8: 97-100. 